\title{
Osteogenesis imperfecta, non-accidental injury, and temporary brittle bone disease
}

\author{
Roger Smith
}

The correct distinction between osteogenesis imperfecta and non-accidental injury (NAI) is an emotive subject upon which the fate of a child can depend. It has been the subject of strongly worded papers and letters by experts. ${ }^{1-5}$ Although the frequency, clinical features, and skeletal effects of NAI are not in question, much new knowledge on osteogenesis imperfecta has bypassed the general paediatrician. ${ }^{67}$ In contrast the position of a temporary fragility of the infantile skeleton related to copper deficiency has, despite new evidence, yet to be established. ${ }^{8}$

\section{(1) Osteogenesis imperfecta}

\section{A. CAUSE}

Impressive advances in our understanding of this syndrome emphasise its clinical and biochemical diversity. ${ }^{67}$ Osteogenesis imperfecta is due to mutations in the genes for type I collagen; a non-functional allele for the alpha 1 (I) chain (gene COLIA1, chromosome 17) halves collagen synthesis and is largely responsible for the mild dominantly inherited form of osteogenesis imperfecta (type I); and single base mutations in the codon for glycine - which occurs in every third residue of the repetitive Gly X Y alpha chain - cause lethal (type II) osteogenesis imperfecta by wrecking formation of the collagen triple helix. Progressive deforming (type III) and type IV osteogenesis imperfecta are less dramatic outcomes of similar glycine mutations in either the alpha 1 (I) or the alpha 2 (I) (gene COLIA2, chromosome 7) chains. Less frequent collagen gene mutations causing skeletal failure delete exons, cause rearrangements, and disturb splice sites.

Not all patients with osteogenesis imperfecta fit comfortably into the Sillence (type I to IV) classification, and likewise not all mutations explain the phenotype ${ }^{9}$; but enough is now known about its clinical and biochemical features to distinguish most cases with some confidence from NAI.

Nuffield Orthopaedic Centre and John Radcliffe Hospital, Headington, Oxford

Correspondence to: Dr Roger Smith, Nuffield Orthopaedic Centre, Windmill Road, Headington, Oxford OX3 7LD.

\section{B. DIAGNOSIS}

\section{(i) Clinical}

In most children the diagnosis of osteogenesis imperfecta is obvious. Type I typically declares itself with a positive family history, blue sclerae, sometimes early onset deafness, and occasional dentinogenesis imperfecta, hypermobility and bruisability; type II by early death; and type III by progressive and later bizarre deformity, scoliosis, sclerae whose blueness fades with age, extreme disability, and short stature. Type IV often causes diagnostic trouble. The sclerae are of normal colour, the dominant family history may be absent, and early radiographs can be normal.

Provided that the niceties of history taking and examination are observed (and often they are not) type I osteogenesis imperfecta should not be missed; but in infancy the radiographs (below) may be normal and in new mutations there will be no family history. Strangely type IV osteogenesis imperfecta and even progressive deforming osteogenesis imperfecta may rarely not declare themselves in the first year; and, for instance, the diagnosis may only be considered when the discoloured teeth of dentinogenesis imperfecta appear. ${ }^{10}$ Recent reviews emphasise the overlap between type III and IV osteogenesis imperfecta, and suggest that type IV is more frequent than previously thought.

\section{(ii) Radiographs}

The gross changes described in subjects with osteogenesis imperfecta who have had fractures are as much due to fractures and their treatment (including immobilisation) as to osteogenesis imperfecta itself. There is nothing in the infantile skeleton that excludes osteogenesis imperfecta. Apart from type II (where the diagnosis is not in question) the radiographs may rarely appear normal in the first year of life, even in infants who later may rapidly develop the obvious features of type III and type IV osteogenesis imperfecta. Likewise no type of fracture excludes osteogenesis imperfecta, and it is possible, although uncommon, for an infant with osteogenesis imperfecta to sustain any type of fracture in a skeleton that is radiologically otherwise normal. In contrast wormian bones are frequent but not universal in all forms of osteogenesis imperfecta.

These comments do not describe the normal appearances in type III are often very severe at birth - with multiple fractures of the ribs and long bones. They merely emphasise that infantile radiological normality does 
not exclude osteogenesis imperfecta. This normality can extend to bone density; radiographs frequently show osteopenia (although bone density is difficult to assess on plain films) but measured forearm bone mineral density can be normal, at least in the adult. ${ }^{11}$

\section{(iii) Biochemistry}

By current techniques collagen abnormalities can be found by experts in up to $85 \%$ of patients with osteogenesis imperfecta. ${ }^{67}$ This important figure influences the decision whether or not to investigate collagen synthesis by dermal fibroblasts where the diagnosis is in doubt. It is important to understand which main abnormalities are being sought; a reduction in the formation of the alpha 1 chain of type I collagen, and the detection of abnormal, additional, or mutant alpha chains. Reduction of the alpha 1 chain is detected by comparison with the alpha chain of type III collagen (which is not altered) expressed as a ratio and compared with collagen synthesised by normal fibroblasts from age matched normal subjects. This quantitative result may be obviously abnormal; but in some subjects with undoubted type I osteogenesis imperfecta the ratio is within the normal range. Far more convincing is the qualitative abnormality due to an abnormal mutant chain. This will show up on appropriate gels either as a clear additional mutant chain or as an obvious widening and slowing of the affected chain due to excessive post-translational modification (further details are provided in papers by Byers $^{67}$ ). This means that apparently normal collagen synthesis by dermal fibroblasts does not exclude osteogenesis imperfecta, but in many patients with osteogenesis imperfecta there is an undoubted collagen abnormality. Such abnormalities are not seen in the normal population. Examples of the usefulness of such biochemical studies are given by Gahagan and Rimsza who describe three children (age 12 and 16 months and 9 years) in whom the initial diagnosis was NAI, subsequently found to have collagen gene mutations. ${ }^{12}$

\section{(2) Non-accidental injury}

Much has been written about NAI, with a large measure of agreement. ${ }^{13}$ Certain features are thought to be highly specific. These include fractures of the metaphyses, ribs, scapulae, vertebrae, the outer ends of the clavicles, bilateral fractures, fractures of different ages, complex fractures of the skull and injuries of the fingers in non-walking children. A controversy continues about the significance of metaphyseal fractures within a normal skeleton; although they strongly suggest NAI they do not appear to be pathognomic of NAI (as some would suggest) and can be seen in osteogenesis imperfecta. Likewise skull fractures can occur in osteogenesis imperfecta.

It is often stated that a lack of explanation for the fractures observed helps the radiologist to diagnose $\mathrm{NAI}^{1}$; this is erroneous since it is in undiagnosed osteogenesis imperfecta that fractures are most often inexplicable.

\section{(3) Temporary brittle bone disease}

Paterson et al recently described 39 patients with fractures occurring only in the first year of life - predictably diagnosed as osteogenesis imperfecta if fractures occurred in hospital and NAI if they did not - in which group rib fractures, metaphyseal abnormalities, and periosteal reactions were common. ${ }^{8}$ Since the numerous fractures healed after the first year, the disorder was considered temporary; this meant that the prognosis was far better than would be expected for a patient with osteogenesis imperfecta with multiple fractures at this age and also raised a question about its cause. While the existence of this disorder seems not to be in doubt (although its apparently high frequency may be) the suggestion that it is due to transient copper deficiency has little scientific support, a fact the authors accept. This suggestion is based on certain similarities to the bone disease of established copper deficiency ${ }^{14}$ and the possibility that such deficiency could interfere with post-translational processing and cross link formation of collagen possibly via copper dependent enzymes such as lysyl oxidase. ${ }^{15}$

In their 39 infants plasma copper estimation at the time of fracturing was obtained in only three ${ }^{8}$; in one ( 8 weeks old) it was undetectable while in two (twins) the concentrations were normal at the same age. From a survey of preterm infants in a special baby care unit Paterson et al suggest that hypocupraemia is more common than currently recognised. ${ }^{8}$ They also found that low plasma copper concentrations could occur in such infants who were apparently thriving; but the possible relationship of copper deficiency to temporary brittle bone disease must still be sub judice.

\section{(4) Practical points}

The features and diagnosis of obvious NAI and osteogenesis imperfecta are agreed upon and provide no difficulty; likewise the frequency of NAI and the infrequency of osteogenesis imperfecta (incidence of about one in 20000 ) is not in doubt. But we are here concerned with the rare but troublesome situation of the infant with unexplained fractures likely to be due to NAI in which the diagnosis of osteogenesis imperfecta has been suggested (for whatever reason). Since any type of fracture can occur in both and be equally inexplicable, and since the skeleton can appear radiologically normal at birth in both (apart from the fractures) there is clearly room for controversy. There are two practical measures which can be taken; first is to examine collagen synthesis from dermal fibroblast culture and second to await events, since an uncertain diagnosis of osteogenesis imperfecta often becomes rapidly obvious after infancy.

Gahagan and Rimsza give useful advice. ${ }^{12}$ They point out inter alia that in appropriate 
underprivileged circumstances NAI is always assumed as the cause for inexplicable fractures; that the child with osteogenesis imperfecta is not immune from NAI (see Knight and Bennett $\left.{ }^{16}\right)$; that none of their three patients with osteogenesis imperfecta had osteopenia or wormian bones; and that a history incompatible with injury is as typical of osteogenesis imperfecta as NAI. They recommend fibroblast culture in infants or children who present with fractures that suggest NAI - first, if there are no external signs of child abuse (such as bruises or head injury); second if the fracture site is consistent with the history, but the mode of injury seems too minor to have caused a fracture; or third if the child has had fractures in different environments. Since the culture of fibroblasts from skin biopsy and subsequent collagen analysis can take at least three months it is important that (where appropriate) the infant is in a safe place during that time. Apart from providing protection this period is not merely spent in waiting for the collagen result, as important changes in the skeleton can occur so rapidly that the diagnosis of osteogenesis imperfecta is soon no longer in doubt.

\section{(5) Court proceedings}

Expert witnesses may wish to win their case, but this should not be done at the expense of the facts and is not necessarily in the best interests of the child. In cases of NAI the approach should be inquisitorial rather than adversarial. ${ }^{17}$ In an ideal world the view given by the expert should be straightforward, not misleading or biased and well researched; a well balanced and non-partisan view will be more welcome to the court than fixed ideas and an inability to consider all sides of the problem.

\section{(6) Conclusion}

The distinction between NAI and osteogenesis imperfecta is a small and untidy corner of paediatrics. Osteogenesis imperfecta is rare and few people have extensive experience of it; the views of those who have should be taken into account, irrespective of their specialty. Where legally necessary biochemical confirmation of osteogenesis imperfecta should be sought. The suggestion that a form of temporary skeletal fragility is due to copper deficiency requires more investigation.

1 Ablin DS, Greenspan A, Reinhart M, Grix A. Differentiation of child abuse from osteogenesis imperfecta. $A \not f R$ 1990; 154: 1035-46.

2 Ablin DS, Greenspan A, Reinhart M, Grix A. Differentiation of child abuse from osteogenesis imperfecta [Letter]. $A \mathscr{R}$ 1990; 155: 1347 .

3 Paterson CR, McAllion SJ. Differentiation of child abuse from osteogenesis imperfecta [Letter]. $A \mathscr{f} R$ 1990; 155: from oste.

4 Ablin DS, Greenspan A, Reinhart M, Grix A Differentiation of child abuse from osteogenesis Differentiation of child abuse from

5 Carty H. Differentiation of child abuse from osteogenesis

6 imperfecta [Letter]. AfR 1991; 156: 635 . In: Scriver CR, Beaudet AL, Sly WS, Valle D, eds. Th In: Scriver CR, Beaudet AL, Sly WS, Valle D, eds. The metabolic basis of inherited diseas

7 Byers PH. Osteogenesis imperfecta. In: Royce PM,
Steinmann B eds. Connective tissue and its heritable disorders. 1st Ed. New York: Wiley Liss, 1993: 317-50. 8 Paterson CR, Burns J, McAllion SJ. Osteogenesis imperfecta: the distinction from child abuse and the recognition of a variant form. Am $\mathcal{F}$ Med Genet 1993; 45: 187-92.

9 Smith R. Osteogenesis imperfecta. From phenotype to genotype and back again. Int $\mathcal{f}$ Exp Pathol 1994; 75: 233-41.

10 Augarten A, Laufer J, Szeinberg A, Passwell J. Child abuse, osteogenesis imperfecta and the grey zone between them. Ұ Med 1993; 24: 171-5.

11 Paterson CR, Mole PA. Bone density in osteogenesis imperfecta may well be normal. Postgrad Med F 1994; 70: imperfect.

12 Gahagan S, Rimsza ME. Child abuse or osteogenesis imperfecta? How can we tell? Pediatrics 1991; 88: 987-92. 13 Carty HML. Fractures caused by child abuse. $f$ Bone foint Surg $B r$ 1993; 75: 849-57.

14 Shaw JCL. Copper deficiency and non-accidental injury. Arch Dis Child 1988; 63: 448-55.

15 Danks DM. Disorders of copper transport: Menkes disease and the occipital horn syndrome. In: Royce PM, Steinmann B, eds. Connective tissue and its heritable disorders. 1st Ed. New York: Wiley Liss, 1993: 487-505.

16 Knight DJ, Bennett GC. Non-accidental injury in osteogenesis imperfecta: a case report. $\mathcal{F}$ Pediatr Orthop 1990; 10: $542-4$.

17 Williams C. Expert evidence in cases of child abuse. Arch Dis Child 1993; 68: 712-4.

\section{Commentary (1)}

Dr Smith has reopened the debate as to the differential diagnosis of bony disorder in infancy by looking at the advances made, particularly in the understanding of the underlying collagen disorder in osteogenesis imperfecta. Unfortunately the clinical investigation of type IV osteogenesis imperfecta has not been undertaken with similar scientific rigor and a description of this variant is required. ${ }^{1}$

Taitz wrote that if osteogenesis imperfecta occurs in 1/20 000 births and type IV osteogenesis imperfecta occurs in $5 \%$ of this total the occurrence of osteogenesis imperfecta with no family history or blue sclera would be in the order of $1 / 3000000$ births. $^{2}$ One to two per cent of all children will be physically abused, although only $5-10 \%$ will sustain fractures. ${ }^{3}$

The diagnosis of physical abuse requires much more than an opinion of a radiograph or even a fibroblast culture. Abuse may occur in families with organic disorders, hence the requirement to carefully build up the diagnostic jigsaw (as Dr Smith says), but this must also be viewed in the wider social context too. There is also more to be understood in the relationship between the biochemical abnormality of the collagen, clinical disease, and fracture. Adults with collagen abnormality do not necessarily have a past history of fractures and current techniques of investigating collagen disorders are time consuming and offer a research rather than a clinical tool.

Fractures in 'mild' disease are, by definition, associated with weight bearing or trauma - rib, skull, and metaphyseal fractures do not occur spontaneously. ${ }^{4}$ Where metaphyseal fractures have been reported in osteogenesis imperfecta it has been in the context of other skeletal abnormality. ${ }^{5}$

Although the skeleton may be apparently normal, wormian bones are common even in 'mild osteogenesis imperfecta', even if they are not apparent at birth or in the immediate postnatal period.

The question of temporary brittle bones 